\title{
Histopathological and ultrastructural features of Koi herpesvirus (KHV)-infected carp Cyprinus carpio, and the morphology and morphogenesis of KHV
}

\author{
Teruo Miyazaki ${ }^{1, *}$, Yoshitaka Kuzuya ${ }^{1}$, Shinya Yasumoto ${ }^{1}$, Masahiro Yasuda ${ }^{1}$, \\ Tatsuya Kobayashi ${ }^{2}$
}

${ }^{1}$ Graduate School of Bioresources, Mie University, 1577 Kurimamachiya-cho, Tsu, Mie 514-8507, Japan

${ }^{2}$ Fuji Biomedix, 10221 Kobitizawa, Hokuto, Yamanashi 408-0044, Japan

\begin{abstract}
Epizootics of Koi herpesvirus (KHV) cause mass mortalities in koi carp and common carp worldwide. We used a newly developed 'per-gill infection' procedure with live KHV, and then conducted detailed histopathological and ultrastructural studies of KHV-infected cells including an examination of the morphology and morphogenesis of KHV. The primary target of KHV was respiratory epithelial cells of the gill lamellae, and release of virions from infected epithelial cells resulted in a systemic infection affecting the kidney, spleen, heart, brain and liver. The pathognomonic feature of infected cells was the formation of intranuclear inclusion bodies with marginal hyperchromatosis in the nucleus. Within the nucleus, assembly of capsids and nucleocapsids and an increase in filamentous nucleoproteins were evident. Enveloped nucleocapsids budded from the inner nuclear membrane into the perinuclear space. De-enveloped nucleocapsids were translocated in the cytoplasm to be embedded within inclusion bodies where tegumentation of the nucleocapsid occurred. Enveloped virions that had budded into intracytoplasmic vesicles and virions located extracellularly were composed of an electron-dense core, surrounded in turn by the capsid, the tegument and finally an envelope with projections. The morphology and morphogenesis of KHV were the same as those of viruses within the family Herpesviridae.
\end{abstract}

KEY WORDS: Koi herpesvirus $\cdot$ Koi $\cdot$ Common carp $\cdot$ Per-gill infection $\cdot$ Histopathology $\cdot$ Electron microscopy $\cdot$ Capsids $\cdot$ Nucleocapsids $\cdot$ Tegument $\cdot$ Virions

\section{INTRODUCTION}

Koi herpesvirus (KHV) is an emerging virus that infects only the common carp Cyprinus carpio carpio and the koi carp C. carpio koi. It has caused mass mortality with vast economic losses in Japan as well as the USA, Israel, and various European, Asian and African countries since 1998 (Hedrick et al. 2000, Neukirch \& Kunz 2001, Gray et al. 2002, Rukyani 2002, Perelberg et al. 2003, Ronen et al. 2003, Haenen et al. 2004, Pikarsky et al. 2004, Sano et al. 2004, Schlotfeldt 2004, Tu et al. 2004, Ishioka et al. 2005). Retrospective studies also suggest that it had already caused mass mortality in 1997 in Germany (Bretzinger et al. 1999).
Because KHV is an emerging virus causing serious disease, it is the subject of intense research and several groups have undertaken its genetic analysis (Gilad et al. 2003, Gunimaladevi et al. 2004, Bercovier et al. 2005, Soliman \& El-Matbouli 2005). Although the size of the KHV genome is bigger than the known herpesviruses, KHV has been included within the family Herpesviridae and is formally designated as Cyprinid Herpesvirus 3 (CyHV-3) (Waltzek et al. 2005).

Certain histopathological and ultrastructural features of KHV-infected fish have been reported by Hedrick et al. (2000) and Perelberg et al. (2003). However, details on the morphology and morphogenesis of the virus, including the maturation process, have not 
been described. Infectivity trials with KHV by immersion and by cohabitation with infected fish resulted in horizontal transmission (Ronen et al. 2003, Adkison et al. 2005, St-Hilaire et al. 2005). In these previous studies the primary target cells of KHV were assumed to be epithelial cells of the gills and skin. Based on histopathological studies on naturally infected fish in Japan (T. Miyazaki et al. unpubl.), we have established a novel KHV challenge method: the 'per-gill infection' method with live KHV (Yasumoto et al. 2006).

In the present study, we performed experimental infections using this method with KHV. The exposed fish were sampled for examination by light and electron microscopy in order to describe histopathological features of the disease, ultrastructural features of KHV-infected cells, and the morphology and morphogenesis of KHV.

\section{MATERIALS AND METHODS}

KHV isolates and experimental fish. Two KHV isolates, IKC03 from Ibaraki Prefecture, Japan (kindly donated by Dr. Fukuda, Tokyo University of Marine Science and Technology) and NKC03 from Nara Prefecture (isolated by the authors) were used. The KHV isolates were cultured using KF-1 cells (donated by Dr. Hedrick, University of California Davis) according to Hedrick et al. (2000). Common carp (mean body weight, $30 \mathrm{~g}$ ) were obtained from the Ono Limnological Laboratory, National Fisheries University, Japan. All fish were offspring of the same parental fish. Koi carp (mean body weight, $10 \mathrm{~g}$ ) were obtained from a private koi farm in Niigata Prefecture, which has not experienced an outbreak of KHV disease. The fish were kept in tanks with a water-recirculation and filtration system at $24^{\circ} \mathrm{C}$.

Per-gill infection. We attempted a novel infection method, in which each fish was challenged with KHV by inoculating a $100 \mu \mathrm{l}$ suspension of $\mathrm{NKC03}$ or IKC03 directly onto the gills $(50 \mu$ to each side of gills, a total of $100 \mu \mathrm{l})$ in air $\left(23^{\circ} \mathrm{C}\right)$ while fish were anesthetized in a $1.5 \%$ solution of carbamic acid ethyl ester. NKC03 was inoculated at a dose of $10^{3.3}$ TCID $_{50} 100 \mu^{-1}$ ( $\mathrm{n}=5$ of common carp), and IKC03 at $10^{3.2} \mathrm{TCID}_{50} 100 \mathrm{\mu l}^{-1}(\mathrm{n}=10$ of common carp) and at $10^{2.2} \mathrm{TCID}_{50} 100 \mathrm{\mu l}^{-1}$ ( $\mathrm{n}=20$ of koi carp). After treatment, the fish were wrapped in wet paper and maintained in air for 5 min to allow KHV to attach to gill cells at air-conditioned room temperature $\left(23^{\circ} \mathrm{C}\right)$, and then returned to the tanks. One control group ( $\mathrm{n}=10$ of koi carp) was treated with saline instead of KHV. Following inoculation, fish were fed on a pelleted diet and observed for clinical signs of disease at $22^{\circ} \mathrm{C}$ for $21 \mathrm{~d}$.
Histopathology and electron microscopy. Moribund fish were removed from the tank and pieces of gills, heart, liver, spleen, kidney, intestine and brain were fixed in Bouin's fluid and processed for histopathological examination. Tissue sections were stained with Mayer's hematoxylin and eosin (H\&E). In addition, pieces of gills, heart, spleen, kidney and brain were fixed in $70 \%$ Karnovsky's solution, postfixed in 1\% $\mathrm{OsO}_{4}$ and processed for electron microscopy. To confirm KHV infection, samples of gills and kidney were stored at $-80^{\circ} \mathrm{C}$ prior to analysis using a KHV-specific PCR (Yuasa et al. 2005).

\section{RESULTS}

\section{Infection experiments}

In the 3 virus-treated groups, diseased fish were first observed $5 \mathrm{~d}$ post-challenge. They were mostly lethargic while some showed shaking or twitching behavior. Most of the diseased fish displayed erosive lesions in the skin, and some also exhibited exophthalmia or endophthalmia. Many moribund fish were observed by 9 and $11 \mathrm{~d}$ post-challenge, and all fish were moribund or died within $3 \mathrm{wk}$. In all moribund fish examined within 2 wk post-challenge, there was swelling of the gills, trunk kidneys and spleen, and in some fish, the bulbus arteriosus was expanded with blood. In the brain of the fish that exhibited twitching while swimming, expanded blood vessels were observed in the optic lobes, cerebellum and medulla oblongata, indicating circulatory disturbances. The KHV PCR detected KHV-specific DNA in the gills and kidneys of those fish. None of the saline-treated fish became moribund, and all were PCR-negative for KHV.

\section{Histopathological features}

Gill. The principal histopathological changes were observed in gill filaments, where most of the respiratory epithelial cells of the gill lamellae were swollen or vacuolated, and exhibited nuclear degeneration. Nuclear changes included pale coloration, karyorrhexis, and the formation of an intranuclear inclusion body (IIB) that was observed as basophilic material within the nucleus with marginal hyperchromatosis caused by deposition of heterochromatin on the inner nuclear membrane (Fig. 1A). Such affected cells were seen under electron microscopy to be infected with KHV (see below 'Electron microscopic features'). These affected respiratory epithelial cells appeared to be fused with cells of the neighboring lamellae, resulting in lamellar fusion and clubbing of the gill filaments 

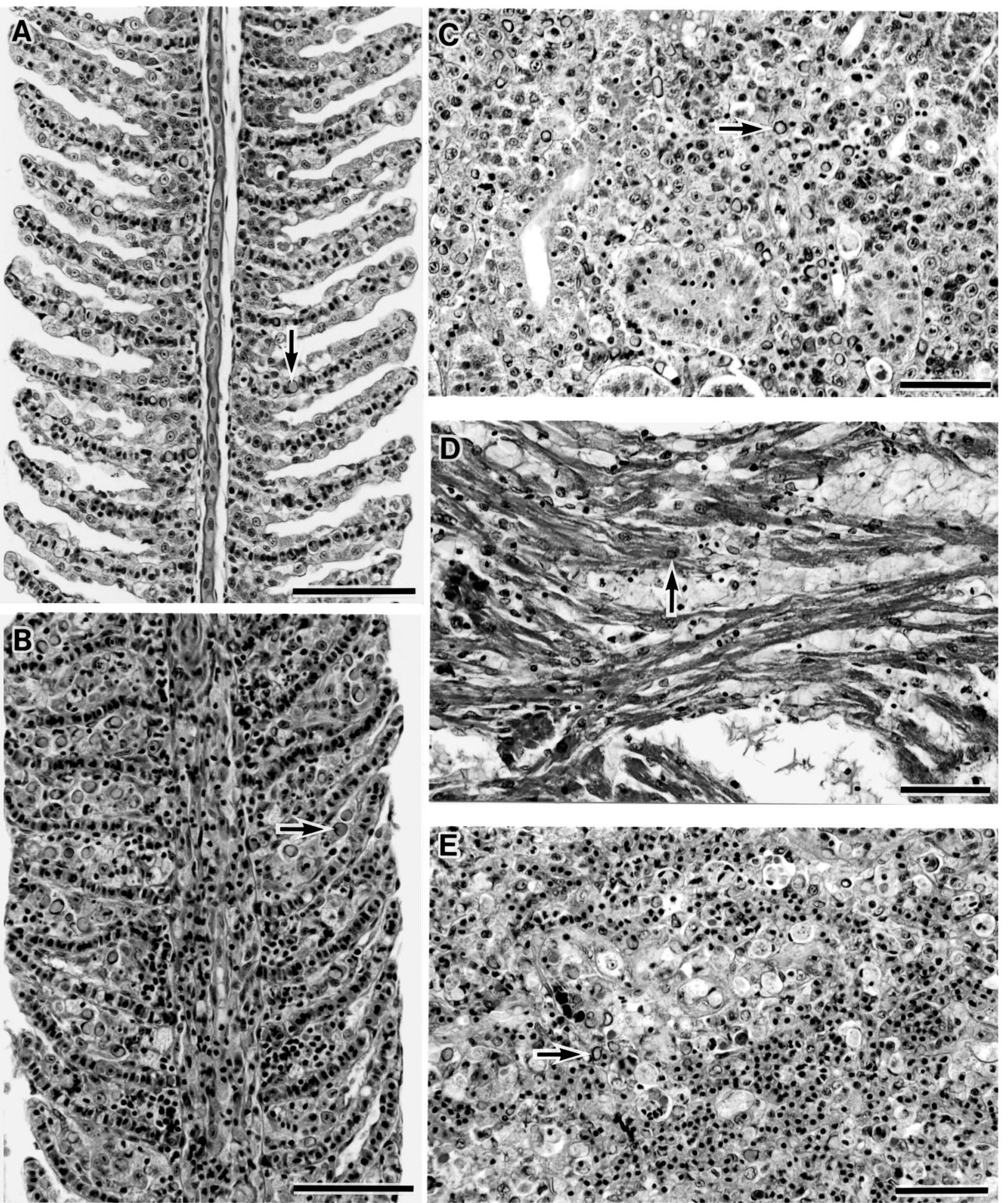

Fig. 1. Cyprinus carpio. Histopathological features of moribund fish (H\&E stain). (A) In the gill filament, respiratory epithelial cells of gill lamellae are swollen with cytoplasmic vacuolation and nuclear degeneration. Some cells are separated. Putative macrophages displaying intranuclear inclusion bodies (IIB) are present within lamellar capillaries. (B) In the gill filament, respiratory epithelial cells of gill lamellae exhibit IIB and are fused with each other. (C) In the kidney, hematopoietic cells are markedly affected exhibiting IIB. Most of the renal tubular epithelial cells have a cloudy appearance. (D) In the ventricle of the heart, many myocardial cells display IIB and have coagulated or dissociated myofibrils. (E) In the spleen, splenocytes in the splenic pulp and ellipsoidal cells in the sheathed artery exhibit IIB. A large number of necrotic swollen cells are present within the splenic pulp and sheathed tissues. Arrows indicate representative cells containing IIB. All scale bars $=30 \mu \mathrm{m}$ 
(Fig. 1B). Necrotic cells were sometimes separated from the respiratory epithelium. Similarly affected cells were observed in the interlamellar epithelium. Cells with IIB were also seen in the lumen of capillaries within the gill lamellae; these appeared to be macrophages (Fig. 1A). Shortly after the peak of incidence of moribund and dead fish in the experiment (hereinafter called the latter period), there was proliferation with karyomitosis of interlamellar epithelial cells, resulting in fusion of gill lamellae. Necrotic cells were seen at the tips of fused lamellae.

Heart. Many myocardial cells exhibited nuclear degeneration (the formation of IIB or pyknosis), and bundles of myofibrils were dilated or coagulated with disappearance of cross-striations (Fig. 1C). In the heart of fish that became moribund during the latter period of the experiment, multiple macrophages and lymphocytes had infiltrated into the affected myocardium.

Kidney. In the kidney, the hematopoietic cells were the main cells affected. In severe cases, many hematopoietic cells were necrotic and many had an IIB and a pyknotic nucleus (Fig. 1D). Most of the tubular epithelial cells exhibited a cloudy appearance and a small number of tubular epithelial cells also showed IIB.

Spleen. IIBs were observed mainly in splenocytes, while ellipsoidal cells were less affected. In severe cases, large numbers of necrotic splenocytes were accompanied by hemorrhage in the splenic pulp (Fig. 1E). Many necrotic cells with acidophilic swollen cytoplasm were also seen among the necrotic splenocytes, but it was not possible to determine whether they were derived from necrotic splenocytes or necrotic macrophages that had migrated into the splenic pulp.

Liver. Only a small number of hepatocytes had IIB. Most hepatocytes exhibited a cloudy appearance.

Brain. In the valvula cerebelli of fish that had exhibited spiral swimming, there was congestion of capillaries and small veins. There were no obvious endothelial lesions in these vessels. Perivasculitis with infiltration of small round cells was seen around some vessels. In the medulla oblongata, IIBs were seen in some small nerve cells (putative internal cells or fasciculus cells) and megalocells.

\section{Electron microscopic features}

In gill filaments, respiratory epithelial cells of the gill lamellae were the main cells infected with KHV as well as epithelial cells in the interlamellar epithelium (Figs. 2 \& 3). In these KHV-infected cells, the assembly of immature capsids and nucleocapsids of KHV was evident in the nucleus (Fig. 2A,C). In the cytoplasm, naked nucleocapsids, tegumented particles within the intracytoplasmic inclusion bodies and membranedelimited structures, and enveloped virions budding into the intracytoplasmic vesicles were observed (Figs. 2A,C,D \& 3A). Moreover, extracellular virions were observed on the cell membrane (Figs. 2C \& $3 \mathrm{~B}, \mathrm{C})$. In affected nuclei, heterochromatin was deposited on the inner nuclear membrane, and there were increased filamentous nucleoproteins and IIBs comprising highly electron-dense granules (Fig. 2A,C). These features were apparent as IIBs by light microscopy. In the nucleus, immature capsids formed first; these were empty round or icosahedral in shape and $100 \mathrm{~nm}$ in diameter. Immature nucleocapsids had an inner core of low electron density. Mature nucleocapsids had an electron-dense core and were circular with a diameter as large as $117 \mathrm{~nm}$. These mature nucleocapsids were usually located within the deposited heterochromatin (Fig. 2A,C). Within the perinuclear space, enveloped mature nucleocapsids (spherical in shape and 150 to $180 \mathrm{~nm}$ in diameter), with narrow envelopes of low electron density, had budded from the inner nuclear membrane (Fig. 2B). Around them the nuclear membranes were deformed and the inner nuclear membrane was damaged, indicating that the mature nucleocapsids had acquired a leaflet of the inner nuclear membrane as the primary envelope when they budded from the nucleus into the perinuclear space. In the cytoplasm, nucleocapsids that had lost the envelope on egress from the perinuclear space (deenveloped nucleocapsids) were translocated not only in the matrix but also in membrane-delimited structures. Moreover, they were embedded in intracytoplasmic inclusion bodies, which were composed of granules of medium electron density and ribosomes, or highly electron-dense granules and membranous structures (Fig. 2C,D). Within the inclusion bodies and membrane-delimited structures, large amounts of tegument material were incorporated into nucleocapsids forming tegumented particles. Tegumented particles were round (183 to $192 \mathrm{~nm}$ in diameter) and displayed a highly electron-dense tegument with a definite surface membrane (Fig. 2B). Inclusion bodies usually contained many tegumented particles while a membrane-delimited structure enclosed one to a few tegumented particles. Virions that became enveloped by budding into cytoplasmic vesicles had the condensed tegument and the thin envelope had surface projections; their diameter was 150 to $167 \mathrm{~nm}$ (Fig. 3A). On the cell membrane, there were extracellularly released virions, which had a thick final envelope with projections. These extracellular virions were 183 to 200 $\mathrm{nm}$ in diameter. The cell membrane in those areas was partially damaged (Fig. 3B,C), indicating that the final envelope of extracellular virions appears to be modified with a leaflet of the plasma membrane. 



Fig. 2. Cyprinus carpio. Electron micrographs of KHV-infected respiratory epithelial cells in gill lamellae. (A) Assembly of immature capsids and nucleocapsids is seen within the nucleus. Heterochromatin is deposited on the inner nuclear membrane and there is an increase in filamentous nucleoproteins. Multiple de-enveloped nucleocapsids have entered the cytoplasm. Tegumented particles are embedded within membrane-delimited structures, inclusion bodies (i) and a vacuole that appears to be derived from a fragmented inclusion body. Scale bar $=1000 \mathrm{~nm}$. (B) A mature nucleocapsid with an electron-dense core is present in the nucleus (n). Two enveloped nucleocapsids (arrows) have budded through the inner nuclear membrane (im) into the perinuclear space (s). A de-enveloped nucleocapsid has entered the cytoplasm. Scale bar $=200 \mathrm{~nm}$. (C) Assembly of immature capsids without a core or nucleocapsids is evident within the nucleus, which displays deposition of heterochromatin on the inner nuclear membrane and intranuclear inclusion bodies (ni). In the cytoplasm, multiple tegumented particles are observed within membrane-delimited structures beside the Golgi apparatus (G). There are large numbers of extracellular virions on the cell membrane. Scale bar $=1500 \mathrm{~nm}$. (D) High-power view of the cell shown in (A). There are many tegumented particles within an inclusion body (i). One tegumented particle is in a membrane-delimited structure (arrow). These indicate that the tegumentation of nucleocapsids takes place within the inclusion body and the membrane-delimited structure. Scale bar $=300 \mathrm{~nm}$ 

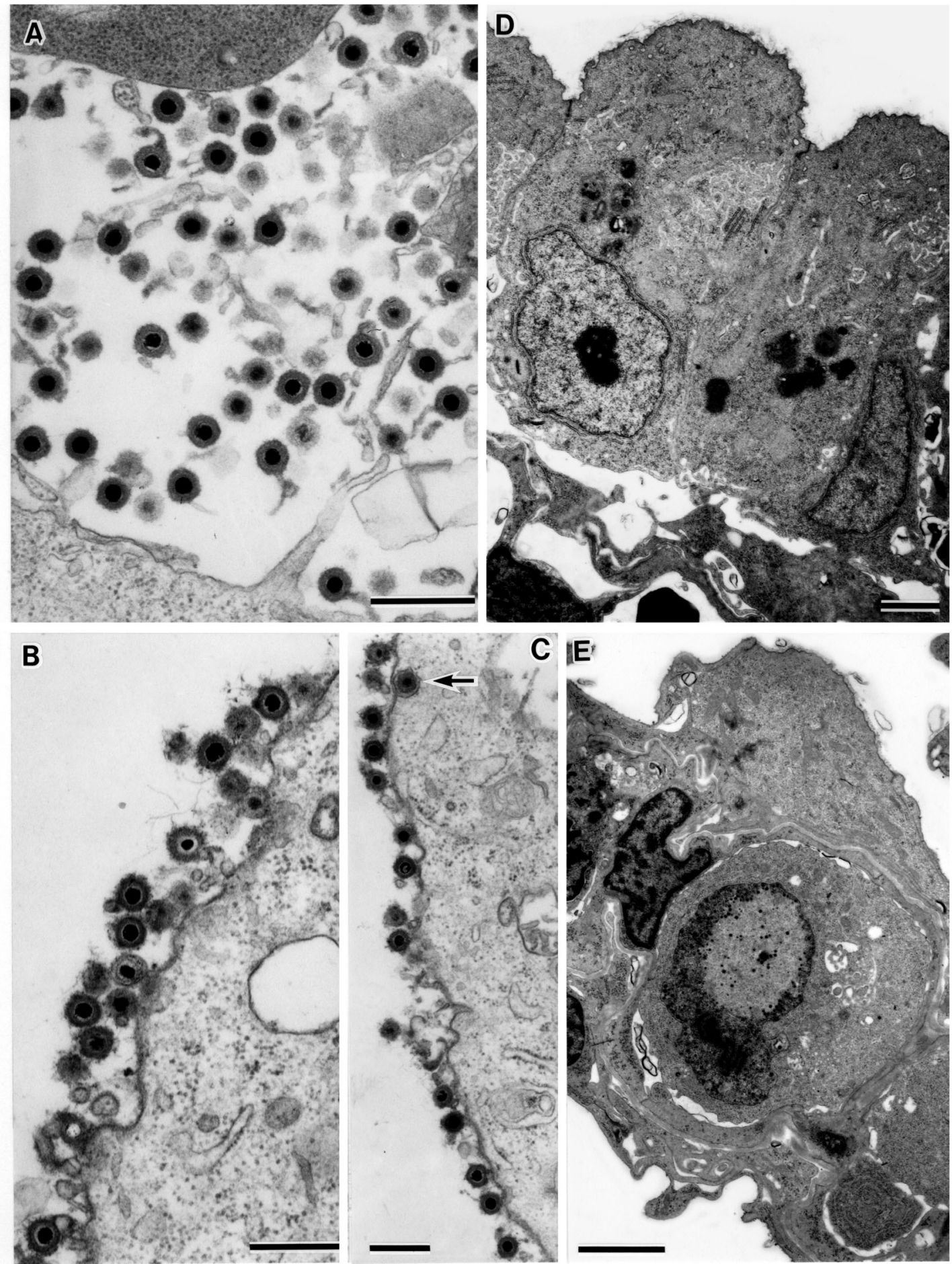

Fig. 3. Cyprinus carpio. (A-C) High-power electron micrographs of respiratory epithelial cells. (A) Many enveloped virions have budded into an intracytoplasmic vesicle. Virions comprise the nucleocapsid, the tegument and a thin envelope with projections. Scale bar $=500 \mathrm{~nm}$. (B,C) Many virions have budded through the cell membrane. These extracellular virions have a condensed tegument and a thick final envelope with projections. In (C), the arrow indicates an enveloped virion transported to the periphery of the cell. Scale bars $=400 \mathrm{~nm}$. (D) Electron micrograph of the respiratory epithelium, which has been partially replaced by cells with a dense cytoplasm containing many mitochondria, rough endoplasmic reticulum, inclusions and developed smooth endoplasmic reticulum. Scale bar $=2000 \mathrm{~nm}$. (E) Electron micrograph of a gill lamella. Within a capillary, there is a macrophage infected with KHV. It is swollen and exhibits assembly of nucleocapsids within the nucleus. Deposition of the heterochromatin, the formation of intranuclear inclusion bodies and an increase in filamentous nucleoproteins are evident. Scale bar $=3000 \mathrm{~nm}$ 
These KHV-infected cells exhibited a dense cytoplasm with normal-looking organelles in the early stage. Assembly of nucleocapsids and an increase in filamentous nucleoproteins were evident in the nucleus. In cells with advanced stages of virus particle maturation, the cytoplasm was electron-lucent and vacuolated, and contained degenerated mitochondria and rough endoplasmic reticulum (rER), and inclusion bodies. Necrotic cells contained no virus particles or fragmented organelles in the clear cytoplasm, and had an electron-lucent nucleus with markedly decreased chromatin and nucleoproteins. With the necrosis of KHV-infected epithelial cells, the respiratory epithelium was replaced by compensatory proliferation of cells, many of which were amoeboid in shape and had a dense cytoplasm containing many mitochondria, rER, inclusions and multiple smooth endoplasmic reticulum (sER) (Fig. 3D).

As well as respiratory epithelial cells, putative macrophages that had migrated into lamellar capillaries were also infected with KHV with assembly of nucleocapsids and virions evident in the nucleus and the cytoplasm, respectively. Virions had also been released into the blood, which could cause a viremia and a systemic infection (Fig. 3E).

In the kidney, hematopoietic cells were markedly infected with KHV with nucleocapsid assembly occurring in the nuclei, which demonstrated marginal hyperchromatosis and increased amounts of filamentous nucleoproteins. Egress of enveloped mature nucleocapsids into the perinuclear space, maturation of viral particles within the cytoplasm, and extracellular virions on the cell membrane were also evident (Fig. 4A). As in the respiratory epithelial cells, tegumentation of de-enveloped nucleocapsids took place within intracytoplasmic inclusion bodies and enveloped virions had budded into intracytoplasmic vesicles in hematopoietic cells. Necrotic cells that had released virions displayed a fragmented nucleus and markedly damaged cytoplasm. Less markedly, renal tubular epithelial cells were also infected with KHV and displayed the same assembly of virus particles.

In the spleen, splenocytes were infected with KHV and they exhibited the same features of assembly and maturation of virus particles within the nucleus and cytoplasm, respectively (Fig. 4B).

Myocardial cells of the heart were infected with KHV. There were assembly of immature capsids and mature nucleocapsids, and an increase in filamentous nucleoproteins within the nucleus, but there were no virus particles in the sarcoplasm (Fig. 4C). The sarcoplasm was electron-lucent, vacuolated, and contained expanded rER, degenerated mitochondria and reduced glycogen. Bundles of myofibrils were fragmented or coagulated with deformation of the intercalated disk.
KHV-infected myocardial cells were infiltrated by lymphocytes, macrophages and granulocytes. Macrophages and granulocytes were also infected with KHV, in which nucleocapsids within the nucleus, maturation of virus particles in the cytoplasm and released virions on the cell membrane were observed (Fig. 4D).

In the medulla oblongata, small nerve cells and megalocells (Fig. 5A) were infected with KHV. Immature capsids and immature nucleocapsids were seen together with an increase in filamentous nucleoproteins within the nucleus. Immature nucleocapsids were mostly embedded within heterochromatin deposited on the nuclear membrane and no egress of enveloped nucleocapsids was observed within the perinuclear space. The perikaryon of KHV-infected nerve cells was slightly damaged and displayed coagulated mitochondria. In the congested capillaries of the valvula cerebelli, there was no evidence of KHV infection in the endothelial cells nor in associated macrophages (Fig. 5B). Surrounding nerve fibers were dissociated by edema.

\section{Assembly and maturation of KHV particles}

Assembly and morphogenesis of herpesvirus particles have been reviewed by Mettenleiter (2002). The morphogenesis of KHV particles was consistent with that report, and is described as follows. Immature capsids and mature nucleocapsids assembled within the nucleus (Figs. 2A-C \& 4). Immature capsids were first formed and were observed as highly electron-dense capsids with a circular or hexagonal outline (Figs. 2C \& $4 \mathrm{C})$. Immature nucleocapsids had an inner core of incompletely organized viral genome of low electron density. Mature nucleocapsids, round or icosahedral in shape, comprised the capsid and an electron-dense core with a completely organized viral genome (Fig. 2A,B). Enveloped mature nucleocapsids had budded from the inner nuclear membrane into the perinuclear space (Figs. 2B \& 4A). The primary envelope was of medium electron density, and appeared to be derived from the inner leaflet of the nuclear membrane.

De-enveloped mature nucleocapsids were observed in the cytoplasm either located in the cytoplasmic matrix (Fig. 2B) or embedded within intracytoplasmic inclusion bodies comprising the ribosomes and granules, or highly electron-dense granules and membranous structures. Tegumentation of de-enveloped nucleocapsid took place within the inclusion bodies and membrane-delimited structures in the cytoplasm (Figs. 2D \& 4A,B,D). Tegumented particles had an electron-dense tegument with a definite surface membrane. Intracytoplasmic inclusion bodies were sometimes destroyed, and several tegumented particles were observed together with fragments of the inclu- 

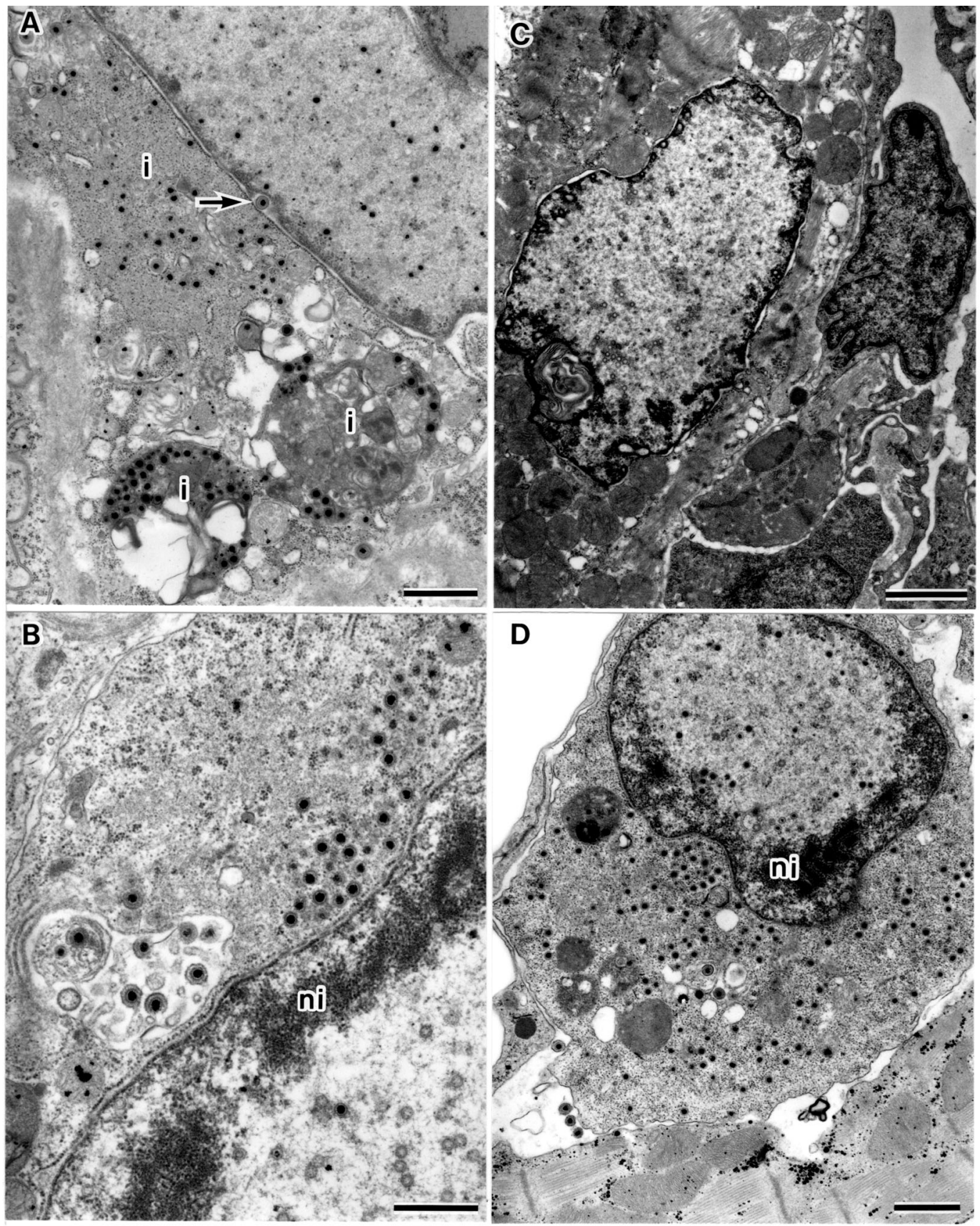

Fig. 4. Cyprinus carpio. Electron micrographs of KHV-infected cells. (A) Hematopoietic cell. In the nucleus, assembly of immature capsids and nucleocapsids, increased filamentous nucleoproteins, and an enveloped nucleocapsid (arrow) budded into the perinuclear space are seen. In the cytoplasm are de-enveloped nucleocapsids and tegumented particles within inclusion bodies (i) comprising fine granules and ribosomes just beside the nucleus, and within other types of inclusion bodies comprising membranous structures or microfilaments. Scale bar $=1000 \mathrm{~nm}$. (B) Splenocyte. In the nucleus, assembly of nucleocapsids, the formation of intranuclear inclusion bodies (ni), and an increase in filamentous nucleoproteins are evident. In the cytoplasm, many naked nucleocapsids are embedded in an inclusion body composed of fine granules beside the nucleus. Enveloped virions have budded into an intracytoplasmic vesicle. Scale bar $=700 \mathrm{~nm}$. (C) Myocardial cell. Assembly of immature capsids and nucleocapsids is evident within the nucleus demonstrating the deformed nuclear membrane. Mitochondria and myofibrils are damaged. Putative lymphocytes have penetrated the infected myocardial cell. Scale bar $=2000 \mathrm{~nm}$. (D) KHV-infected macrophage that has penetrated the myocardial cell. Assembly of immature capsids and nucleocapsids, and the formation of intranuclear inclusion bodies (ni) are seen in the nucleus. There are many naked nucleocapsids in the cytoplasm. A small number of tegumented particles are seen within membrane-delimited structures and an inclusion body. Enveloped virions have been released from the cell. Scale bar $=1000 \mathrm{~nm}$ 

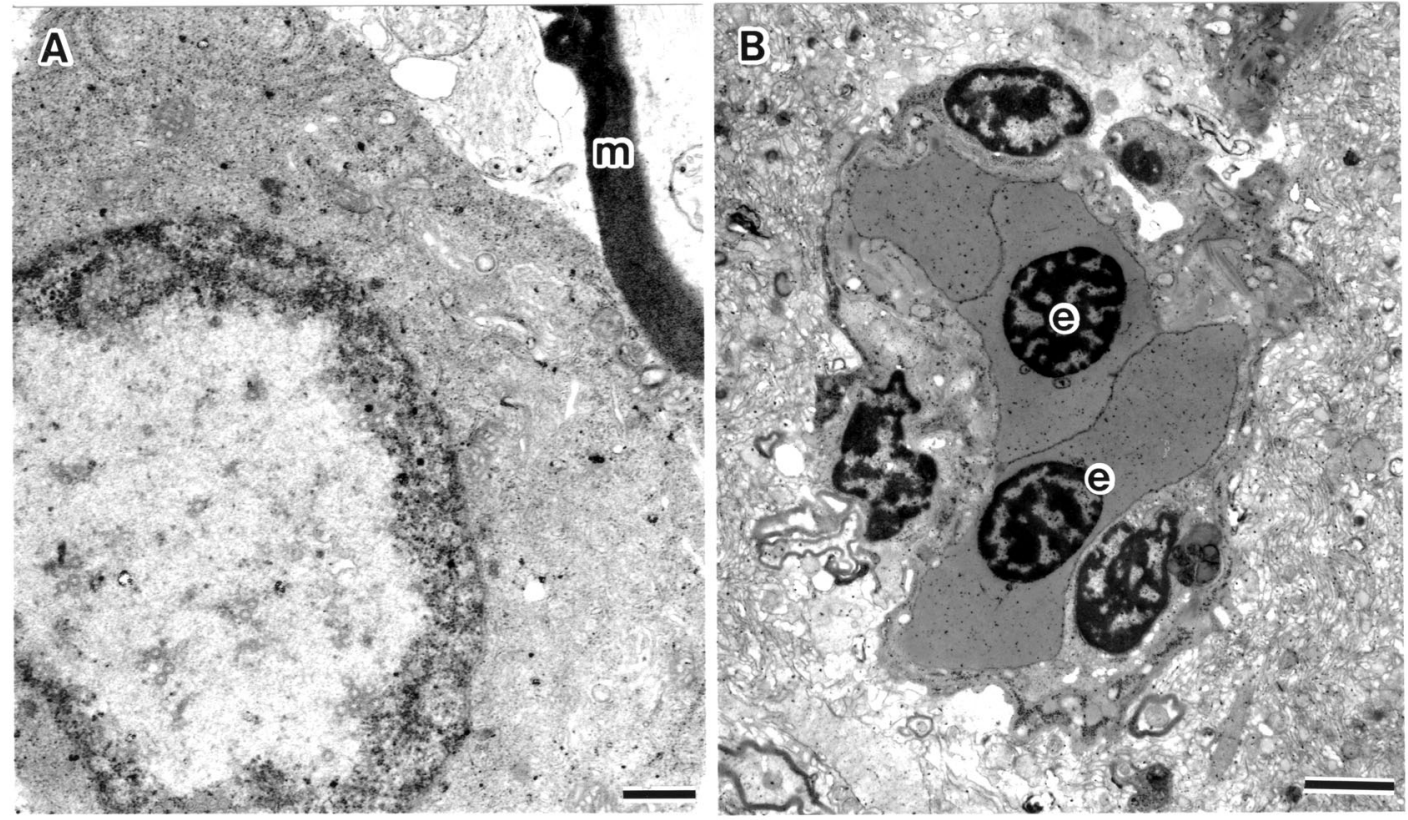

Fig. 5. Cyprinus carpio. Electron micrographs. (A) KHV-infected megalocell in the medulla oblongata. Assembly of many immature capsids and an increase in filamentous nucleoproteins are evident in the nucleus. Immature capsids are mostly embedded within peripherally deposited heterochromatin. The perikaryon is slightly damaged and displays coagulated mitochondria. $\mathrm{m}=$ myelinated nerve fiber. Scale bar $=600 \mathrm{~nm}$. (B) Congested capillary in the valvula cerebelli. There is no evidence of KHV infection in the capillary endothelial cell and putative macrophages within the lumen. One cell has an intracytoplasmic inclusion body and another penetrates the capillary. Around the capillary, nerve fibers are edematously dissociated. e $=$ erythrocytes. Scale bar $=3000 \mathrm{~nm}$

sion bodies (Fig. 2A). Enveloped virions, which had an electron-dense envelope with surface projections on the condensed tegument, budded from the intracytoplasmic membrane into vesicles (Figs. 3A \& 4B). These enveloped virions were 150 to $225 \mathrm{~nm}$ in diameter, which varied according to the infected cell type. Enveloped virions were transported within vesicles to the periphery of the cell (Fig. 3C). Extracellular virions budding from the cell membrane (Fig. 3B,C) had a condensed tegument and a thick final envelope with projections. Their size varied from 167 to $200 \mathrm{~nm}$ in diameter according to the infected cell type.

\section{DISCUSSION}

In the present study, KHV infection of carp was established by a novel 'per-gill infection' method that may simulate a natural route of infection. Using this procedure, the essential histopathological and ultrastructural features of KHV-infected fish were identified. In the per-gill infection, primary target cells were respiratory epithelial cells of the gill lamellae, where nuclear degeneration and IIB were evidence of infection. The formation of IIB was revealed to be due to an increase in filamentous nucleoproteins and the assembly of multiple viral capsids and nucleocapsids. Matu- ration of KHV took place within the cytoplasm and multiple virions were released from the cells, suggesting that the released virions could cause systemic infection. Experimentally, diseased fish externally displayed erosive lesions in the skin that appeared to be due to necrosis of epidermal cells caused by dissemination of KHV via the water environment. In the gill lamellae, lamellar capillaries contained KHV-infected macrophages, in which assembly of virus particles was completed and from which virions were released. This finding indicated that the virions that had propagated within the respiratory epithelial cells entered the capillaries to infect macrophages as well as to cause systemic dissemination via the bloodstream. As a direct result of this viremia, multiple types of cells were infected with KHV: for example, hematopoietic cells and renal tubular epithelial cells in the kidney, splenocytes, myocardial cells, nerve cells, hepatocytes, and macrophages and granulocytes in the blood. Epithelial cells of gills, hematopoietic cells and renal tubular epithelial cells in the kidney, splenocytes, hepatocytes and epidermal cells have been described as major target cells in cases of natural infection as well as experimental infection by bath exposure (Hedrick et al. 2000, Perelberg et al. 2003, Gilad et al. 2004). Thus, KHV infects multiple cell types in carp. Virogenesis was completed in hematopoietic cells, renal tubular epithe- 
lial cells, splenocytes and macrophages, while assembly of nucleocapsids alone was evident in myocardial cells and nerve cells. Thus, KHV-infected fish showed severe necrosis of visceral organs. Diseased fish appear to die due to respiratory disturbances and damage to multiple organs. In addition to KHV, a pox-like virus causing 'Koi sleepy disease' (KSD) also infects respiratory epithelial cells, causing respiratory disturbances (Miyazaki et al. 2005). The resultant hypoxia induced the sleepy behavior (lying on the bottom of an aquarium) of infected fish. In contrast, KHV-infected fish showed lethargy but not sleepy behavior, although they suffered from hypoxia as evident from the damage to the mitochondria in hepatocytes and renal tubular epithelial cells, which had a cloudy appearance by light microscopy. Comparing the pox-like virus and $\mathrm{KHV}$, the former infects only respiratory epithelial cells, while KHV infects multiple cell types in visceral organs as well as respiratory epithelial cells. In KHVinfected fish, perhaps the pain associated with necrosis of many visceral organs accounts for a different clinical syndrome compared with KSD infection.

Diseased fish sometimes displayed abnormal swimming indicating nervous disturbances, and indeed, nerve cells in the medulla oblongata were infected with KHV. Moreover, congestion of capillaries and small vessels was evident in the valvula cerebelli and medulla oblongata. However, there was no evidence that KHV infection of the endothelial cells or migration of KHV-infected macrophages had caused emboli within the lumen. Around the congested blood vessels, edematous dissociation of nerve fibers was observed. This appeared to be a circulatory disturbance resulting from hypoxia and cardiac failure, and induced a subsequent infiltration of inflammatory cells to cause perivasculitis. Damage to nerve cells and congestion with perivasculitis appear to have been associated with nervous disturbances in the medulla oblongata as well as the valvula cerebelli, and may account for the abnormal swimming behavior.

Thus, results obtained by electron microscopy have allowed a full description of the morphology and morphogenesis of KHV. The results confirm that KHV has the same morphology as viruses of the family Herpesviridae (Davison et al. 2005). Moreover, the morphogenesis and maturation steps of KHV were not different from those of other herpesviruses (Mettenleiter 2002). Waltzek et al. (2005) designated KHV as CyHV-3 based on the results of genomic analysis. Our results support their claim.

In summary, isolates of KHV were shown to infect koi carp and common carp experimentally using a pergill method. KHV infected multiple cell types during the systemic stage of infection. Morphology and morphogenesis of KHV were described.
Acknowledgements. We thank Dr. P. F. Dixon (CEFAS Weymouth Laboratory) for his valuable advice, Dr. H. Fukuda (Tokyo University of Marine Science and Technology) for donating an experimental KHV isolate, and Dr. R. P. Hedrick (University of California Davis) for donating KF-1 cells. We also thank Dr. Y. Takahashi and Dr. M. Kondo (Department of Applied Aquabiology, National Fisheries University) and a koi farmer in Niigata Prefecture for providing the experimental carp.

\section{LITERATURE CITED}

Adkison MA, Gilad O, Hedrick RP (2005) An enzyme linked immunosorbent assay (ELISA) for detection of antibodies to the koi herpesvirus (KHV) in the serum of koi Cyprinus carpio. Fish Pathol 40:53-62

Bercovier H, Fishman Y, Nahary R, Sinai S and others (2005) Cloning of the koi herpesvirus (KHV) gene encoding thymidine kinase and its use for a highly sensitive PCR based diagnosis. BMC Microbiol 5:13

Bretzinger A, Fischer-Scherl T, Oumouna M, Hoffman R, Truyen U (1999) Mass mortalities in koi carp, Cyprinus carpio, associated with gill and skin disease. Bull Eur Assoc Fish Pathol 19:182-199

Davison AJ, Eberle R, Hayward GS, McGeoch DJ and others (2005) Herpesviridae. In: Fauquet CM, Mayo MA, Maniloff J, Desselberger U, Ball LA (eds) Virus taxonomy: 8th report of the International Committee on Taxonomy of Viruses. Elsevier, San Diego, p 193-212

Gilad O, Yun S, Adkison MA, Way K, Willits HN, Bercovier H, Hedrick RP (2003) Molecular comparison of isolates of an emerging fish pathogen, koi herpesvirus, and the effect of water temperature on mortality of experimentally infected koi. J Gen Virol 84:2661-2668

Gilad O, Yun S, Zagumutt-Vergara FJ, Leutenegger CM, Bercovier H, Hedrick RP (2004) Concentration of a Koi herpesvirus (KHV) in tissues of experimentally infected Cyprinus carpio koi as assessed by real-time TaqMan PCR. Dis Aquat Org 60:179-187

Gray WL, Mullis L, LaPatra SE, Groff JM, Goodwin A (2002) Detection of koi herpesvirus DNA in tissues of infected fish. J Fish Dis 25:171-178

Gunimaladevi I, Kono T, Venugopal MN, Sakai M (2004) Detection of koi herpesvirus in common carp, Cyprinus carpio L., by loop-mediated isothermal amplification. J Fish Dis 27:583-589

Haenen OLM, Way K, Bergmann SM, Ariel E (2004) The emergence of koi herpesvirus and significance to European aquaculture. Bull Eur Assoc Fish Pathol 24:293-307

Hedrick RP, Gilad O, Yun S, Spangenberg JV and others (2000) A herpesvirus associated with mass mortality of juvenile and adult koi, a strain of a common carp. J Aquat Anim Health 12:44-57

Ishioka T, Yoshimizu M, Izumi S, Suzuki K and others (2005) Detection and sequence analysis of DNA polymerase and major envelope protein genes in koi herpesvirus derived from Cyprinus carpio in Gunma prefecture, Japan. Vet Microbiol 110:27-33

> Mettenleiter TC (2002) Herpesvirus assembly and egress. J Virol 76:1537-1547

> Miyazaki T, Ishiki T, Hori K (2005) Histopathological and electron microscopy studies on sleepy disease of koi Cyprinus carpio koi in Japan. Dis Aquat Org 65:197-207

Neukirch M, Kunz U (2001) Isolation and preliminary characterization of several viruses from koi (Cyprinus carpio) suffering gill necrosis and mortality. Bull Eur Assoc Fish Pathol 21:125-135 
Perelberg A, Smirnov M, Hutoran M, Diamant A, Bejerano Y, Kotler M (2003) Epidemiological description of a new viral disease affecting cultured Cyprinus carpio in Israel. Isr J Aquacult 55:5-12

Pikarsky E, Ronen A, Abramowitz J, Sivan BL and others (2004) Pathogenesis of acute viral disease induced in fish by Carp Interstitial Nephritis and Gill Necrosis Virus. J Virol 78:9544-9551

Ronen A, Perelberg A, Abramowitz J, Hutoran M and others (2003) Efficient vaccine against the virus causing a lethal disease in cultured Cyprinus carpio. Vaccine 21: 4677-4684

Rukyani A (2002) Koi Herpesvirus infection in Indonesia. Dis Info 15

Sano M, Ito T, Kurita J, Yanai T, Watanabe N, Miwa S, Iida T (2004) First detection of koi herpesvirus in cultured common carp Cyprinus carpio in Japan. Fish Pathol 39: 165-167

Schlotfeldt HF (2004) Severe losses of common carp in Germany due to Koi Herpesvirus (KHV). Bull Eur Assoc Fish Pathol 24:216-217

Editorial responsibility: Mark Crane, Geelong, Victoria, Australia
Soliman H, El-Matbouli M (2005) An inexpensive and rapid diagnostic method of Koi Herpesvirus (KHV) infection by loop-mediated isothermal amplification. Virol J 2:83

St-Hilaire S, Beevers N, Way K, Deuff RML, Martin P, Joiner C (2005) Reactivation of koi herpesvirus infections in common carp Cyprinus carpio. Dis Aquat Org 67:15-23

Tu C, Weng MC, Shiau JR, Lin SY (2004) Detection of koi herpesvirus in koi Cyprinus carpio in Taiwan. Fish Pathol 39:109-110

Waltzek TB, Kelley GO, Stone DM, Way K and others (2005) Koi herpesvirus represents a third cyprinid herpesvirus (CyHV-3) in the family Herpesviridae. J Gen Virol 86:1659-1667

Yasumoto S, Kuzuya Y, Yasuda M, Yoshimura T, Miyazaki T (2006) Oral immunization of common carp with a liposome vaccine fusing koi herpesvirus antigen. Fish Pathol 41:141-145

Yuasa K, Sano M, Kurita J, Ito T, Iida T (2005) Improvement of a PCR method with the SphI-5 primer set for the detection of koi herpesvirus (KHV). Fish Pathol 40:37-39

Submitted: May 18, 2007; Accepted: April 29, 2008

Proofs received from author(s): June 13, 2008 